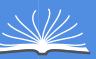

Global journals Inc.

है

\title{
Influence of the Volume of the Ischemic Origin on Clinical Outcomes of the Brain Stroke
}

\author{
By Z. A. Akbarkhodjaeva \& G. S. Rakhimbaeva \\ Tashkent Medical Academy
}

Abstract- In this article authors discussed about the influence of volume of the ischemic origin on clinical outcomes in patients with stroke. Cerebral ischemic stroke is one of the main cause of death among cardiovascular and brain diseases. The study is dedicated to learn the relationship of the dynamics of the volume of the ischemic focus with clinical outcome of stroke. For this study, 125 patients were examined and analyzed. MRI of the brain in acute period of ischemic stroke in $78 \%$ of patients were assessed that foci of ischemia of small (less than $10 \mathrm{~cm}^{3}$ ), medium $\left(10-50 \mathrm{~cm}^{3}\right.$ ) and large size (more than $50 \mathrm{~cm}^{3}$ ). Lacunar strokes, as well as the size of the penumbra, affecting the ability to restore impaired brain functions, can be identified only by magnetic resonance imaging of the brain.

Keywords: ischemic stroke; cerebral disease; ischemic origin; MRI; heart attack.

GJMR-A Classification: NLMC Code: WL 348

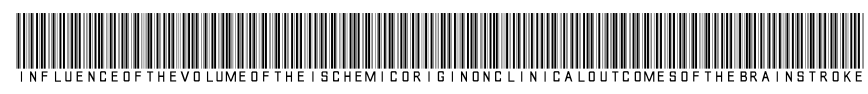

Strictly as per the compliance and regulations of:

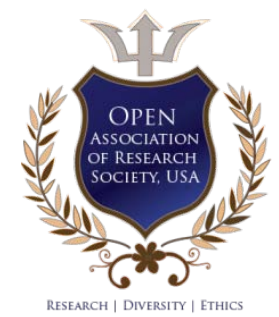

(C) 2019. Z. A. Akbarkhodjaeva \& G. S. Rakhimbaeva. This is a research/review paper, distributed under the terms of the Creative Commons Attribution-Noncommercial 3.0 Unported License http://creativecommons.org/licenses/by-nc/3.0/), permitting all noncommercial use, distribution, and reproduction in any medium, provided the original work is properly cited. 


\title{
Influence of the Volume of the Ischemic Origin on Clinical Outcomes of the Brain Stroke
}

\author{
Z. A. Akbarkhodjaeva ${ }^{\alpha} \&$ G. S. Rakhimbaeva ${ }^{\circ}$
}

Abstract- In this article authors discussed about the influence of volume of the ischemic origin on clinical outcomes in patients with stroke. Cerebral ischemic stroke is one of the main cause of death among cardiovascular and brain diseases. The study is dedicated to learn the relationship of the dynamics of the volume of the ischemic focus with clinical outcome of stroke. For this study, 125 patients were examined and analyzed. MRI of the brain in acute period of ischemic stroke in $78 \%$ of patients were assessed that foci of ischemia of small (less than $10 \mathrm{~cm}^{3}$ ), medium $\left(10-50 \mathrm{~cm}^{3}\right)$ and large size (more than $50 \mathrm{~cm}^{3}$ ). Lacunar strokes, as well as the size of the penumbra, affecting the ability to restore impaired brain functions, can be identified only by magnetic resonance imaging of the brain.

Keywords: ischemic stroke; cerebral disease; ischemic origin; MRl; heart attack.

\section{INTRODUCTION}

$\mathrm{C}_{\mathrm{s}}$ erebral ischemic strokes, leading to severe clinical, expert and social consequences, occupy a special place in terms of their significance among cerebral vascular diseases [2, 4]. At the present stage of development of technical capabilities of medicine, it is advisable to determine prognostic criteria using a combination of clinical and instrumental methods of research $[1,3]$.

A large role is also played by the size, localization of the lesion, the presence or absence of perifocal edema $[4,5]$. There is practically no data in the literature on the possible relationship between the size of the focus of a stroke and the outcomes in the course of long-term follow-up, especially in terms of restoring impaired functions. At the same time, such data will make it possible to realistically predict the possibilities of rehabilitation over a relatively long period, which is very important not only from a clinical, but also from an economic point of view.

The purpose of the study is to determine the relationship of the dynamics of the volume of the ischemic focus with the clinical outcome of cerebral stroke.

\section{Materials and Methods}

125 patients undergoing cerebral ischemic stroke (IS) was examined, studied and analyzed. Age grading ranged from 40 to 90 years. Among all surveyed

Author a $\sigma:$ Department of Neurology, Tashkent Medical Academy, Tashkent, Uzbekistan.e-mail: author.uzb@mail.ru women, there were 40\% (50 patients), men did not significantly prevail and amounted to $60 \%$ (75 patients).

Taking into account the design of the examination, all 125 patients in the acute period of stroke (2- th $^{\text {th }}$ day from the onset of the disease) underwent magnetic resonance imaging (MRI) of the brain and also from 28 days to 6 months from the onset of the disease re-performed MRI of the brain the brain.

\section{Results}

In most cases, the left middle cerebral artery was affected (50\%), then the vertebrobasilar basin (27\%) and the right middle cerebral artery (23\%).

The volume of a large focus was more than 50 $\mathrm{cm}^{3}$, the middle focus was from 10 to $50 \mathrm{~cm} 3$, and small ones were related to foci with a volume of up to $10 \mathrm{~cm} 3$. The category of small ones included heart attacks in the vertebrobasilar basin and the lacunar strokes identified by us. In most cases, the hemodynamic stroke subtype was found (47.2\%). Lacunar stroke against arterial hypertension was registered in $22.4 \%$ of cases.

Among the subtypes of heart attacks, the following were verified: extensive heart attack (9), deep stem (11), cortical and subcortical heart attack (4), small cortical (4), lacunary heart attack (29).

According to the data obtained, patients with verified extensive cerebral infarction also had the most severe degree of neurological deficit. According to all assessment scales, these patients were associated with severe neurological deficit, which corresponded to a pronounced and constant dependence of patients on the nearest social environment.

After a course of inpatient treatment, these patients only slightly improved their manifestations of the disease.

Patients who had a stem, but not extensive heart attack, were also characterized by the presence of a pronounced neurological deficit, corresponding to severe and moderate severity according to the estimated scales. After the course of inpatient treatment, there was no significant increase in scores and the transition on the scales to the category of less severe motor disorders, but there was a decrease in the degree of dependence on others according to the Barthel scale at $\mathrm{P}<0.01$.

Similar data on the dynamics of recovery were obtained in groups of patients who had a lesion of medium and small size in the subcortical structures of 
the brain. Patients from these groups within the same subsection of the assessment of the degree of neurological deficit improved their performance, without going into the group with a less severe degree of motor impairment. At discharge from the hospital, patients from these groups had only a moderate, approaching mild, degree of dependence on the nearest social environment.

Note that in our study, the hyposensitive lesion included not only the core of ischemia, but also penumbra. In this regard, in the acute period of $\mathrm{Al}$, a quantitative assessment of the neurological deficit included neurological disorders, caused in general by a hypo-intensive focus. In part, this can also explain the much better and larger amount of recovery of impaired functions in case of $\mathrm{Al}$ in the acute period of inpatient treatment for large lesions, compared with small lesions.

Thus, the quantitative indices of the neurological deficit in the acute period of Al depended on the volume of the primary lesion (with penumbra), the vascular basin, and the type of stroke.

In the acute period with different volumes of ischemia and stroke types, the recovery period from the acute and 22-24 days was characterized by an increase in self-service functions in the range of 20-30 points according to the Barthel index and regression of focal symptoms 2-5 points on the NIHSS scale.

The best recovery of impaired functions was observed with medium sizes of the ischemic focus, and the smallest - with small lesions of ischemia.

Comparison of the rates of recovery of neurological disorders in dynamics showed that in the acute period of $\mathrm{Al}$, the regression of the neurological deficit occurred at least 2 times more actively.

The initiation of treatment during the "therapeutic window" was also of great importance in restoring lost functions and shaping the size of the ischemic focus.

73 people from 125 patients, inpatient treatment was started in the first 6 hours after the onset of Al. The later dates for the start of inpatient treatment worsened the effectiveness of remedial measures. Thus, out of 49 patients with $42 \%$ of patients with primary or secondary, high-volume ischemia, 42 patients started treatment later than 6 hours after the onset of stroke.
Thus, there is no doubt that the intensity of the recovery period in the acute stage of cerebral stroke is influenced (along with other factors) by the size of the lesion and the localization of the infarction of the brain, proximity to the motor vehicle is especially important.

However, comparison of the clinical picture in the acute phase of stroke with the localization and size of the lesion does not reveal a direct correlative connection, in particular, central hemiplegia and hemianesthesia occur with extensive foci of ischemia, and with limited lacunar infarctions in the deep.

We analyzed the group of patients (65 people) who had positive dynamics of the disease after 2 years from the moment of stroke, to assess the factors that determine a positive outcome, namely the severity of the patients' condition in the early period, the age of the patients at the time of the disease, the size and the location of the lesion, if any, according to the MRI data, was provided, the mood for treatment of $e$ and adherence to secondary prevention by the main factors.

All patients from this group had a mild or minimal neurological deficit at the time of admission to the hospital, a fairly young age (45-49 years old - 22 people, 50-59 years old - 43 people), when surveyed 49 people out of 65 had a positive attitude towards treatment and punctual secondary prevention.

A positive result was obtained in those subjects who did not have a severe neurological deficit at the onset of the disease, had a commitment to the secondary prevention of recurrent strokes and had no signs of depression.

When analyzing the indicators in this group examined after 3 years, there was an increase in the number of recurrent strokes, a decrease in the number of patients with positive dynamics due to an increase in the severity of somatic pathology (GB, CHD, atherosclerosis, diabetes), ignoring the rules for secondary prevention of strokes, and weighting of neurological deficit in patients with extensive primary lesion of the brain.

To determine the indicators that affect the recovery process, both in the immediate and in the longterm period, a correlation analysis was made of the factors identified in the early recovery period of cerebral stroke with remote outcomes after 1-3 years (Table 1).

Table 1: Factors affecting the long-term outcomes of rehabilitation treatment for strokes and their correlation

\begin{tabular}{|c|c|c|}
\hline \multirow{2}{*}{ Factors to be considered } & \multicolumn{2}{|c|}{ Correlation indicator } \\
\cline { 2 - 3 } & After 2 years & After 3 years \\
\hline Weight & $-0,59$ & $-0,36$ \\
\hline Age & $-0,32$ & $-0,38$ \\
\hline Volume of hearth & $-0,49$ & $-0,51$ \\
\hline Blood Pressure & $-0,16$ & $-0,20$ \\
\hline Diabetes & 0,26 & $-0,31$ \\
\hline Gender & 0,09 & 0,06 \\
\hline
\end{tabular}


Taking into account our analysis after 2 years, the relationship is maintained with such factors as age, localization of the lesion, the state of the emotional sphere. After 3 years, the effect of somatic pathology weighting in the form of coronary heart disease and diabetes mellitus increases.

Thus, the prediction of recovery of impaired functions is not affected by the factor of gender. Such factors as stabilization of concomitant pathology improve the prognosis while observing the rules of secondary prophylaxis and adherence to the recommended therapy; positive emotional mood, struggle with anxiety and depression, good physical activity before the onset of the disease and regular, targeted physical therapy exercises after a stroke.

The prognosis is worsened by the extensiveness of the primary focus of the brain and its location in the older age groups at the time of stroke. Of course, the size of the primary focus is not always correlated with the outcome of the disease, but among other factors, this relationship is most obvious. However, working with positive factors to some extent can help the patient cope with the disease, despite the vastness of the primary focus.

\section{FINDINGS}

1. Using MRI of the brain in the acute period of cerebral stroke in $78 \%$ of patients revealed foci of ischemia of small (less than $10 \mathrm{~cm} 3$ ), medium (10-50 cm3) and large size (more than $50 \mathrm{~cm} 3$ ). Lacunar strokes, as well as the size of the penumbra, affecting the ability to restore impaired brain functions, can be identified only by magnetic resonance imaging of the brain.

2. The size of the lesion during ischemic cerebral stroke plays an important, but not the only role in taking into account the restoration of impaired functions. In $46 \%$ of patients with cerebral strokes, a direct correlation was found between the magnitude of the focus of the stroke and its clinical and functional manifestations.

3. When performing neuroimaging in the acute period of ischemic cerebral stroke, it is advisable to determine the size of the primary lesion and the state of the penumbra, which makes it more likely to predict the recovery dynamics of impaired functions.

\section{References Références Referencias}

1. Ershov V. I. The most acute period of ischemic stroke: clinical and pathogenetic characterization, prognosis, optimization of neuroprotective therapy: abstract of thesis. ... Doctor of medical sciences. M., 2011. - $45 \mathrm{p}$.

2. Prediction of early neurological deterioration using diffusion- and perfusion-weighted imaging in hyper acute middle cerebral artery ischemic stroke// Arenillas J. F, Rovira A, Molina C A, et al.//Stroke. 2002; 33: 2197-2205.

3. Atrial fibrillation as an independent predictor for no early ecanalization after iv-t-PA in acute ischemic stroke //Kimura K, Iguchi Y, Yamashita S, et al. //J Neurol Sci 2008; 267: 57-61.

4. Acute ischemic stroke update. //Baldwin K, Orr S, Briand $M$ et al. //Pharmacotherapy 2010; 30: 493-14.

5. Campbell B C, Mitchell $P \mathrm{~J}$, Kleinig $T \mathrm{~J}$, et al. Endovascular therapy for ischemic stroke with perfusion-imaging selection. N Engl J Med. 2015; 372(11): 1009-1018. 